\title{
Linx
}

Revue des linguistes de l'université Paris X Nanterre

9 | 1997

Émile Benveniste. Vingt ans après

\section{Pour une sémantique de l'art}

\section{Gérard Dessons}

\section{(2) OpenEdition \\ Journals}

Édition électronique

URL : http://journals.openedition.org/linx/1077

DOI : 10.4000/linx.1077

ISSN : 2118-9692

\section{Éditeur}

Presses universitaires de Paris Nanterre

\section{Édition imprimée}

Date de publication : 1 avril 1997

Pagination : $327-333$

ISSN : 0246-8743

\section{Référence électronique}

Gérard Dessons, «Pour une sémantique de l'art », Linx [En ligne], 9 | 1997, mis en ligne le 09 juillet 2012, consulté le 19 avril 2019. URL : http://journals.openedition.org/linx/1077 ; DOI : 10.4000/ linx. 1077 


\title{
Pour une sémantique de l'art
}

\author{
Gérard Dessons
}

$\mathrm{C}$ omme l'indique son titre, mon intervention a le sens d'une proposition : le concept de sémantique, central dans la théorie du langage de Benveniste, me semble une voie pertinente pour l'exploration de ce qu'on a appelé la « question de l'art». Bien que la notion d'art occupe une place relativement marginale dans les Problèmes de linguistique générale - dont ce n'est pas, effectivement, l'objet -, elle remplit, dans l'étude "Sémiologie de la langue », une fonction stratégique.

Ce qui m'intéresse, dans le concept de "sémantique », ce sont les deux valeurs qu'il a chez Benveniste : à la fois la valeur «technique » d'un mode de signifiance particulier, et celle, de portée générale, qui ajuste la théorie du langage à une signification de l'expérience, avec le sens d'une "sémantique générale ", comme celle que vise Benveniste dans son projet de "culturologie», lorsqu'il évoque "une sémantique qui passe à travers tous [1]es éléments de culture et qui les organise ${ }^{1} »$.

C'est donc dans l'étude de 1969, «Sémiologie de la langue », que la relation entre l'art et la signification est abordée. Bien qu'il s'agisse ici spécifiquement des arts plastiques, la réflexion pourra être étendue aux autres arts, comme la musique, dont parle aussi Benveniste, et la littérature, qui est l'artlangage, dont il ne parle pas, mais vers laquelle convergent pourtant ses analyses. On peut même penser que le discours que tient Benveniste sur les arts plastiques est le discours qu'il n'a pas tenu sur la poésie, dont je rappelle qu'il la jugeait «immensément » intéressante pour la linguistique ${ }^{2}$. A ce titre, l'étude "Sémiologie de la langue » porte en creux le fondement d'une poétique qu'il n'a pas faite. C'est en tout cas à propos de l'art, et plus exactement des

\footnotetext{
1 «Structuralisme et linguistique », Problèmes de linguistique générale II, Gallimard, 1974, (abrégé ensuite en PLG II), p. 25.

2 «Ce langage qui fait l'histoire », PLG II, p. 37.
} 


\section{Gérard Dessons}

«expressions artistiques ${ }^{3}$ », que l'idée d'une "sémantique sans sémiotique » (p. 65) est formulée, posant l'autonomie d'un concept qui désigne un mode spécifique de signification dissocié du mode sémiotique.

Le problème posé par Benveniste est de savoir comment l'art signifie, et pour cela, il faut savoir ce qui, en art, dans les pratiques signifiantes considérées du point de vue de l'art, signifie. Là se pose la question, déterminante, de l'unité. A partir de la musique, Benveniste montre qu'un art peut reposer sur des unités, en l'occurrence, les notes, sans pour autant qu'elles soient des signes. Mais c'est avec les arts plastiques que le problème de l'unité pose de la façon la plus radicale le problème du sémantique. Tout d'abord, à la différence de la musique, les unités n'y apparaissent pas spécifiques d'une pratique : pour la peinture ou le dessin, l'unité est-elle « la figure, le trait, la couleur ?4 » Mais, surtout, en se demandant si «la question, ainsi formulée, a [...] encore un sens », Benveniste met en cause la pertinence de toute unité plastique en dehors de l'œuvre dont elle est une composante : « Les relations signifiantes du "langage" artistique sont à découvrir à l'intérieur d'une composition » (p. 59).

L'unité sémantique se définit donc contrastivement avec l'unité sémiotique, le signe qui, non seulement transcende les réalisations individuelles, mais se présente comme un élément discret, alors que le mode sémantique se caractérise par la nature continue de ses unités. Ce qui, par parenthèse, implique l'irréductibilité de la poésie comme art aux signes qui la composent.

C'est pourquoi, confondre la signifiance d'une œuvre plastique avec la désignation lexicale de ses composantes a pour conséquence non seulement la sortie hors du système de l'œuvre, mais surtout, l'introduction de la discontinuité dans un système dont aucune unité n'est discrète. C'est le sens du propos de Braque sur les spectateurs qui réduisent leurs commentaires à l'inventaire des objets représentés sur les toiles: "Ces gens-là ont l'air d'ignorer totalement que ce qui est entre la pomme et l'assiette se peint aussi ${ }^{5}$ ». En faisant de ce qu'il appelle l'« entre-deux » un «élément aussi capital » que ce que les spectateurs « appellent l'objet », Braque pensait le tableau comme un continu, en-dehors de la nomination des figures représentées, qui ne peut isoler des unités qu'en faisant abstraction de la spécificité de l'objet plastique, en l'occurrence, d'être un objet peint.

Dans un tableau, on ne sait a priori ni où commence ni où finit une unité. Dès qu'on désigne par un nom - et donc qu'on circonscrit - ce que Benveniste appelle une "entité formelle $e^{6}$, on sort du système sémantique du tableau, dans la mesure où on l'informe d'une sémiotique qui appartient à la langue. $\mathrm{Si}$ l'on prend l'exemple de la couleur, Benveniste fait remarquer qu'en tant que

3 «Sémiologie de la langue », PLG II, p. 65.

4 «Sémiologie de la langue », PLG II, p. 56.

${ }^{5}$ Entretien de Georges Braque avec Georges Charbonnier, L'Express, 2 juillet 1959. Cité par Michel Vinaver dans la Présentation de Iphigénie Hôtel, Actes Sud, 1993, p. 13.

6 «Sémiologie de la langue», PLG II, p. 56. 
matériau, elle "comporte une variété illimitée de nuances gradables, dont aucune ne trouvera d'équivalence avec un "signe" linguistique » (p. 59). Cette constatation pose implicitement le problème de la relation de la peinture et du langage: comment parler de la peinture, si le système de la langue est inadéquat aux systèmes des œuvres plastiques?

Picturalement, les couleurs sont les unités d'un système chaque fois spécifique, puisque c'est «dans la composition seule qu'elles s'organisent et prennent, techniquement parlant, une "signification", par la sélection et l'arrangement » (p. 58). En soulignant le terme signification, Benveniste ne fait pas allusion aux systèmes chromatiques, préexistants, des théories plastiques, mais bien à quelque chose qui se passe à l'intérieur de chaque œuvre d'art : « La signifiance de l'art ne renvoie donc jamais à une convention identiquement reçue entre partenaires. Il faut en découvrir chaque fois les termes, qui sont illimités en nombre, imprévisibles en nature, donc à réinventer pour chaque œuvre » (p. 59-60).

La mise en avant de la singularité des œuvres, de leur primat sur tout système plastique ou esthétique général ${ }^{7}$, énonce la spécificité de l'art : "L'art n'est jamais ici qu'une œuvre d'art particulière» (p. 59). Avec l'irréductibilité des œuvres, c'est la subjectivité qui se trouve placée au cœur même de la question de l'art. Devant une œuvre d'art, il ne s'agit plus «de conditions générales et constantes, mais d'une caractéristique individuelle» (p. 57). C'est en ce sens que « l'artiste crée [...] sa propre sémiotique » (p. 58).

Cette spécificité de l'œuvre d'art, qui définit sa signification par l'affirmation de sa subjectivité, c'est exactement ce qu'on appelle la manière. concept à réinventer, la manière nomme indissociablement une forme et l'historicité d'une pratique ${ }^{8}$. Je reprends l'exemple de la couleur. La caractérisation d'une couleur peut se faire de différentes façons : en référence à une matière (bleu de cobalt), à un objet du monde (jaune citron), mais elle peut aussi référer à une manière ; ce que montrent, dans les nuanciers des couleurs à l'huile, les dénominations "rouge Angelico», «brun Van Dyck», «vert Véronèse ». Ces appellations sont exemplaires du mode sémantique, en ce qu'elles montrent que dans un tableau, ce n'est pas $d u$ vert qui est un élément de la signifiance, mais un vert particulier, dont la valeur est tributaire du système-sujet de l'œuvre. Ce que désigne le "vert Véronèse » est indissociablement une couleur et une manière. Je ne parle pas du matériau vendu sous ce nom ; analysé en composants chimiques, il ne renvoie plus qu'indirectement à la pratique qui l'a inventé. Je veux souligner la singularité d'une appellation qui fait référence à une œuvre, et donc à une historicité.

Le processus est sans doute plus significatif dans l'appellation «bleu Patinir ", notoire, bien que non enregistrée sur les nuanciers de couleurs, non

\footnotetext{
${ }^{7}$ Le « fonctionnement des systèmes dits artistiques » est considéré « en ignorant délibérément leur fonction esthétique » (ibid., p. 58).

8 Pour un exposé de la question, je renvoie à mon article «Les enjeux de la manière", dans Langages, $n^{\circ} 118$, juin 1995, p. 56-63.
} 


\section{Gérard Dessons}

«matérialisée ». Qu'est-ce qu'un «bleu Patinir »? C'est un bleu qu'on trouve sur les toiles du paysagiste flamand, une couleur qui est ce qu'elle y est. On peut certainement la décrire, c'est-à-dire tenter d'en analyser les composantes chromatiques, mais ce mouvement est infini, puisqu'une teinte, Benveniste l'a souligné, n'est pas une entité discrète. De plus, le «bleu Patinir », renvoyant à une manière, n'est pas qu'une teinte : il est aussi une façon d'être associé à des formes, à d'autres couleurs, une façon d'accrocher la lumière, une touche.

Dans le texte de Benveniste, la nature psychologique, intentionnelle, d'un sujet-artiste qui «choisit » ses couleurs et "les dispose à son gré sur la toile » (p. 58) peut faire problème. Mais ce point est relativement négligeable, puisque la mise en avant de la subjectivité dans la peinture, même sous cet aspect traditionnel, ouvre finalement sur la formulation d'une théorie du sémantique dans la langue comme "ordre [qui] s'identifie au monde de l'énonciation et à l'univers du discours » (p. 64), un ordre qui, précisément, met

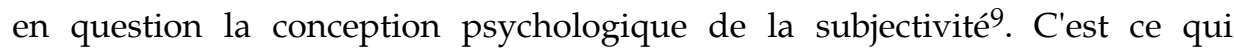
explique que "signifier », du point de vue du mode sémantique, ne réfère pas à un " sens ", mais, radicalement, à une situation : "Le sens de la phrase implique référence à la situation de discours, et l'attitude du locuteur ${ }^{10}$ »; ce qui revient à rendre indissociables le sujet, l'histoire et le langage.

Dans « Sémiologie de la langue », en 1969, Benveniste poursuit et précise le travail commencé trois ans auparavant dans "La forme et le sens dans le langage ». En 1966, il définissait la phrase comme l'« expression du sémantique », pour la raison qu'elle «n'est que particulière ${ }^{11}$ », contrairement au signe, pour lequel « tout ce qui est individuel est exclu» (p. 223). Il apparaît que, tout en élaborant sa conception du sémantique à partir de l'art, c'est bien dans le langage qu'il en trouve les conditions de théorisation. Il est significatif, de ce point de vue, que dans son approche de l'art il ait besoin d'une terminologie linguistique, même s'il en souligne le caractère métaphorique, contre l'usage courant, qui, lui, oublie la métaphore. Benveniste n'implique pas, en effet, que l'art soit lui-même un langage ${ }^{12}$, puisque la «double signifiance » dont la langue est investie reste « un modèle sans analogue » (p. 63).

En fait, on ne peut lire les propos théoriques sur l'art, objet indirect de la théorie de la double signifiance, en les isolant d'un autre concept : la fonction d'interprétance de la langue, qui rend la signification de l'art inséparable de la médiation du langage à travers le système de la langue. $\mathrm{Si}$, d'une part, «la langue est nécessairement l'instrument propre à décrire, à conceptualiser, à interpréter tant la nature que l'expérience » (p.96), et si, d'autre part, elle

\footnotetext{
9 Voir «De la subjectivité dans le langage», Problèmes de linguistique générale, Gallimard, 1966, (abrégé ensuite en PLG I), p. 260.

10 « La forme et le sens dans le langage », PLG II, p. 225.

11 «La forme et le sens dans le langage», loc. cit.

$12 \mathrm{Au}$ sens où «le langage se réalise nécessairement dans une langue » («Remarques sur la fonction du langage dans la découverte freudienne », PLG I, p. 85).
} 
« fonde la possibilité de tout échange et de toute communication, par là de toute culture » (p.60), alors, une pratique artistique, à l'image de la société, « devient signifiante dans et par la langue » (p. 96).

Que l'art implique le langage comme interprétant, cela signifie que le langage est le seul système qui permette de faire de l'art la subjectivitéhistoricité d'une pratique signifiante. Le langage fonde la représentation, la mimèsis, au sens d'Aristote dans la Poétique, c'est-à-dire non pas la copie, la duplication du monde, sur le modèle platonicien, mais le processus par lequel l'expérience du monde se trouve informée et historicisée par une subjectivité, et qui, en transformant les pragmata en muthos, réalise une véritable " sémantique du monde » liant signification et subjectivation.

La théorie du sémantique, comme théorie de la signifiance de l'art, élaborée à partir d'une conception discursive du langage, montre ce que ne montrent pas les théories de l'image ; précisément parce que leur objet n'est pas l'art, mais l'image, et que le mode sémiotique peut alors y fonctionner. C'est une chose de reconnaître qu'une publicité pour les pâtes Panzani renvoie, comme Roland Barthes l'écrivait en 1964, au «signifié esthétique » de la nature-morte; c'en est une autre d'analyser une nature-morte comme une publicité de pâtes. Et s'il est sans doute légitime de considérer qu'une image publicitaire fonctionne comme un signe ou un ensemble de signes, la méthode de la nomination-désignation, qui en épuise alors la description ${ }^{13}$, ne peut que manquer la signifiance d'une œuvre d'art, qui, elle, est continue. L'art échappe au signe, parce que le signe ne dit rien de cette subjectivité-langage qui, par l'activité d'interprétance, informe l'objet dans l'expérience artistique.

C'est ce que montre, dans les descriptions de tableaux, l'utilisation des formes déictiques de la langue, qui constituent la situation d'énonciation en référence absolue, c'est-à-dire fondatrice, du système de l'œuvre. La deixis est organisatrice de la vision et de son objet, dans le rapport à un je parlant et voyant tout à la fois. Le langage fait voir, dans la mesure où il institue en présences les choses vues, qui, dès lors, apparaissent comme des extentions du présent du discours. C'est le sens même de l'adjectif praesens, tel que l'analyse Benveniste : "Ce qui est praesens [...] n'est pas séparé par un intervalle du moment où l'on parle. [...] Dès lors praesens s'applique à ce qui est "sous les yeux, visible, immédiatement présent" ${ }^{\prime \prime}$ ».

Ainsi, les déictiques spatiaux et le présent de l'indicatif instituent en point de vue un point de parole, comme dans la description d'une Tempête de Vernet par Diderot: «Là, des matelots secourent un malheureux sans vêtements [...]. Ici, une femme échappée de la fureur des flots est entraînée loin

\footnotetext{
13 « Des paquets de pâtes, une boîte, un sachet, des tomates, des oignons, des poivrons, un champignon, le tout sortant d'un filet à demi ouvert, dans des teintes jaunes et vertes sur fond rouge », (R. Barthes, « Rhétorique de l'image », Communications, n 4, 1964, p. 40-41).

14 «Le système sublogique des prépositions en latin », PLG I, p. 135.
} 


\section{Gérard Dessons}

d'eux par des mains secourables ${ }^{15}$ ». Tout le travail de Diderot montre que pour regarder un tableau, il faut l'écrire. Cet enjeu majeur des Salons, que représentent les relations entre langage et peinture, Louis Marin le repère, résumant le projet de Diderot de cette façon: "Il faudrait que l'écriture décrivante fasse voir le tableau ${ }^{16}$ ». Mais, s'intéressant aux "pouvoirs de l'image » plus qu'à la question de l'art, il fait du «poème iconique » (p. 90) de Diderot l'écriture d'un tableau remémoré : «Il transcrit le soir, la nuit, le lendemain, sa mémoire d'objets "absents" » (p. 73). Ce qui est en cause, dans l'idée de cette écriture qui se souvient, c'est la conception de la représentation, mise du côté de la reproduction. Alors que ce que montre la tentative de Diderot, c'est que l'écriture du tableau n'est pas la reproduction d'une expérience visuelle antérieure, mais l'accession de cette expérience au statut d'expérience artistique. Le temps de l'art est le temps du discours de l'art, le temps de l'interprétation, qui institue une pratique en art, et ses objets en œuvres. ${ }^{17}$ Le processus artistique peut être décrit dans les termes mêmes qu'utilise Benveniste à propos de l'interprétation freudienne: "Les événements empiriques n'ont de réalité pour l'analyste que dans et par le "discours" qui leur confère l'authenticité de l'expérience ${ }^{18}{ }^{\prime}$.

Une sémantique de l'art, impliquant l'instanciation d'un sujet dans et par son discours, suppose une poétique. L'expérience d'une manière plastique se fait dans et par une manière de langage, un «style », pour reprendre encore la terminologie qu'emploie Benveniste à propos de la signifiance des rêves: «C'est dans le style, plutôt que dans la langue, que nous verrions un terme de comparaison avec les propriétés que Freud a décelées comme signalétiques du "langage" onirique » (p. 86). La notion de style forme ici, avec la notion de langue, un couple conceptuel dont il faut souligner l'importance. Comme le montre le contexte, c'est à partir du concept de discours que le concept de style est introduit dans la réflexion, Benveniste évoquant des "comparaisons fécondes entre la symbolique de l'inconscient et certains procédés stylistiques du discours » (p. 86). En opposant une logique du style à celle de la langue, il confère à une notion "littéraire», relevant donc d'un domaine considéré en général comme extérieur à celui d'une linguistique "pure», le statut de concept opératoire dans sa théorie de la signification.

Même si Benveniste se réfère à la conception traditionnelle du style - celle, rhétoricienne, des figures : "L'inconscient use d'une véritable "rhétorique" qui, comme le style, a ses "figures" et le vieux catalogue des tropes fournirait un inventaire approprié aux deux registres de l'expression »-, l'opposition entre les concepts de langue et de style ouvre le champ de la linguistique à celui de

\footnotetext{
15 Diderot, Salon de 1771, dans CEuvres esthétiques, Garnier, 1968, p. 583.

16 L. Marin, Des pouvoirs de l'image : Gloses, Le Seuil, 1993, p. 76.

17 J'ai développé cette question du temps de l'œuvre d'art dans un article intitulé «Rythme, genèse, peinture ", Ritm, n 9, 1994, p. 29-37.

18 «Remarques sur la fonction du langage dans la découverte freudienne », PLG I, p. 77.
} 
la poétique, puisqu'il s'agit de désigner, à propos du rêve, un mode de signification propre à la littérature et, plus particulièrement, à la poésie: «Certaines formes de poésie peuvent s'apparenter au rêve» (p. 83). Cette signifiance se trouve en creux dans le "suspens du sens », compris comme un "mode de structuration » différent de celui de la langue. L'opposition entre langue et style, en face des dichotomies: langue et parole, langue et discours, préfigurait donc, en 1956, l'opposition de 1969 entre sémiotique et sémantique. Quant aux « analogies » (p. 86) que Benveniste soulignait entre l'expérience onirique et l'expérience littéraire, elles s'expliquent par le fait que, toutes spécificités maintenues, il s'agit chaque fois, comme également dans l'expérience artistique, d'interpréter du subjectif, c'est-à-dire, dans les termes d'Henri Meschonnic, du continu et de l'inconnu.

L'inconnu, dans une œuvre d'art, tient à cette "sémiotique » personnelle, qui est indissociablement une matière et une manière, une matière informée de manière qui la structure et la rend signifiante. Ce que le discours interprétant poursuit, c'est «la manière dont l'inventeur d'un style façonne la matière commune»(p. 87), et il le fait à sa manière. Autrement dit, le sujet devient, par son discours, sujet de l'art en devenant linguistiquement cette aventure suscitée par un objet qu'il reconnaît informé de manière. Dire la manière devrait être l'enjeu même du discours de l'art, que ce dire prenne, dans une œuvre littéraire, la forme d'un poème, ou, dans un exposé technique, la forme d'une expression, d'une formule ${ }^{19}$.

Pour une sémantique de l'art, considérer un objet comme une œuvre d'art, c'est avant tout reconnaître qu'il signifie. Non qu'il a un sens, mais qu'il a $d u$ sens, pour nous. Et cette signifiance, postulée a priori, n'a de réalité que d'être la matérialisation d'une "vision ", pour reprendre le terme de Benveniste 20 , avec la spécificité d'être à la fois une forme et un sujet. Cela explique que l'art, étant producteur de valeurs, soit irréductible à l'analyse par le mode sémiotique, parce que ce qui fait d'un tableau, fût-il figuratif, un objet d'art excède la totalité de ses signes. Mais, étant du subjectif, cet excès - et en cela une sémantique de l'art est critique d'une phénoménologie de l'art - n'est ni un indicible (seul le signe fait le sacré en transformant en ineffable ce qui échappe à sa logique), ni un imperceptible, car une manière se perçoit doublement: comme entité et comme identité.

\footnotetext{
${ }^{19}$ Voir G. Dessons, «Dire la manière: les poèmes de peintres ", Le texte, l'cuvre, l'émotion, Bruxelles : La Lettre volée, 1994, p. 215-226.

20 «L'artiste n'[a] ni de "réponse" à attendre, ni de contradiction à éliminer, mais seulement une vision à exprimer » («Sémiologie de la langue », PLG II, p. 59). La position de Benveniste est sur ce point très proche de celle de Proust dans une page célèbre du Temps retrouvé : "Le style pour l'écrivain aussi bien que la couleur pour le peintre est une question non de technique mais de vision. Il est la révélation, qui serait impossible par des moyens directs et conscients, de la différence qualitative qu'il y a dans la façon dont nous apparaît le monde " (A la recherche du temps perdu, Gallimard, coll. « La Pléiade », t. IV, 1989, p. 474).
} 